\section{Predicting Period-Doubling Bifurcations and Multiple Oscillations in Nonlinear Time-Delayed Feedback Systems}

\author{
Daniel W. Berns, Jorge L. Moiola, and Guanrong Chen
}

\begin{abstract}
In this brief, a graphical approach is developed from an engineering frequency-domain approach enabling prediction of perioddoubling bifurcations (PDB's) starting from a small neighborhood of Hopf bifurcation points useful for analysis of multiple oscillations of periodic solutions for time-delayed feedback systems. The proposed algorithm employs higher order harmonic-balance approximations (HBA's) for the predicted periodic solutions of the time-delayed systems. As compared to the same study of feedback systems without time delays, the HBA's used in the new algorithm include only some simple modifications. Two examples are used to verify the graphical algorithm for prediction: one is the well-known time-delayed Chua's circuit (TDCC) and the other is a time-delayed neural-network model.
\end{abstract}

Index Terms - Harmonic-balance approximation, Hopf bifurcation, period-doubling bifurcation, time-delayed feedback.

\section{INTRODUCTION}

In the last two decades, there has been continuously increasing interest in studying the coexistence of periodic and chaotic behaviors in nonlinear dynamical systems, such as some electronic devices and physiological organisms. Interdisciplinary research among specialists with different backgrounds from applied and computational mathematics, circuits and systems engineering, physical and biomedical sciences, etc., has been highly motivated and, recently, has become very active. In particular, research on analysis of dynamical behaviors of a living or artificial neural network (modeled by nonlinear circuits) has attracted much attention from the scientific and engineering communities.

In a (living or artificial) neural network, the special feature of multiple oscillations is a mechanism that has been recognized as important for memory storage. Therefore, detecting the coexistence of periodic and chaotic behaviors in a general nonlinear dynamical system has a great impact on understanding and utilizing complex networks; for this purpose, an efficient prediction algorithm is essential.

Still, investigating a complex system with multiple equilibria, periodic solutions, and particularly chaotic behaviors is a real challenge. In order to carry out qualitative analysis, it is preferable to have simple models that possess such complicated, but typical features. For instance, it has been suggested [1], [2] to use simple electronic circuits as basic prototypes for biological systems suffering the socalled dynamical disease [3]-[6] or to use simple mechanical systems [7] to characterize some biological and engineering processes. A notable distinctive characteristic of such systems is the existence

Manuscript received January 10, 1997; revised February 6, 1998. The work of D. W. Berns was supported by UNPSJB. The work of J. L. Moiola was supported by CONICET (the National Council of Scientific Research of Argentina) and by Fundación Antorchas. The work of G. Chen was supported by the U.S. Army Research Office under Grant DAAG55-98-1-0198. This paper was recommended by Associate Editor T. Endo.

D. W. Berns is with the Departamento de Electrónica, Universidad Nacional de la Patagonia San Juan Bosco, 9000 Comodoro Rivadavia, Argentina.

J. L. Moiola is with the Departamento de Ingeniería Eléctrica, Universidad Nacional del Sur, 8000 Bahía Blanca, Argentina.

G. Chen is with the Department of Electrical and Computer Engineering, University of Houston, Houston, TX 77204-4793 USA.

Publisher Item Identifier S 1057-7122(98)05306-9. of time delays in the system models, typically in a feedback form. These delays commonly occur as a consequence of finite conduction time in one of the several system variables. This time-delayed characteristic leads to a more complicated mathematical description using difference-differential or functional-differential equations for the underlying systems. It has been observed that simple circuits, even those described by scalar difference-differential equations, can show very complex and subtle dynamics [8]. Representative circuits of this type include the first-order model of a phase-locked loop with time delays [9] and the well-known time-delayed Chua's circuit (TDCC) [10]. These two families of simple circuits both have chaotic dynamics, and can be used in many applications such as laboratory models for living neural networks, vehicles for chaos and bifurcations control, and devices for secure communications.

The purpose of this brief is to show how the analysis of simple time-delayed systems can be carried out by using engineering frequency-domain techniques and harmonic-balance approximations (HBA's). In so doing, formulation of a general setting for timedelayed systems studied in [11] is further extended to handle difference-differential equations of the neutral type. A simple algorithm is then developed for predicting the appearance of period-doubling bifurcations (PDB's) starting close to Hopf bifurcation points. The TDCC is used to test and verify the new algorithm. In addition, the capacity of the proposed graphical-analysis method is applied to the detection of multiple oscillations in a time-delayed neural-network model frequently discussed in the literature [12], [13].

\section{Harmonic-Balance Principle for Time-Delayed NONLINEAR FEEDBACK SYSTEMS}

Consider the following parametrized time-delayed nonlinear difference-differential equation of the neutral type:

$$
\begin{aligned}
\dot{x}(t-\tau)+A_{0}(\mu) \dot{x}(t)= & A_{1}(\mu) x(t)+A_{2}(\mu) x(t-\tau) \\
& +B(\mu) g[C(\mu) x(t-\tau) ; \mu] \\
y(t)= & C(\mu) x(t)
\end{aligned}
$$

where $A_{0}, A_{1}$, and $A_{2}$ are $n \times n$ matrices, $B$ is an $n \times r$ matrix, $C$ is an $m \times n$ matrix, $\mu \in R$ is the system bifurcation parameter, $y \in R^{m}$ is the system output, the smooth nonlinear function $g: R^{m} \rightarrow C^{2 q+1}\left(R^{r}\right)$ can be viewed as a system input $u \in R^{r}$, $\tau>0$ is a time-delay constant, and " $q$ " is the degree of smoothness necessary for a $(2 q+1)$ th-order Taylor series expansion of the nonlinear function, $q=1,2,3,4$.

Taking Laplace transforms on both sides of (1) gives

$$
\begin{aligned}
{\left[s\left(\exp (-s \tau)+A_{0}\right)-A_{1}-A_{2} \exp \right.} & (-s \tau)] X(s) \\
= & B \mathcal{L}\{g[C x(t-\tau) ; \mu]\}
\end{aligned}
$$

which can be rewritten as

$$
\begin{aligned}
X(s)=\left[s\left(\exp (-s \tau)+A_{0}\right)-A_{1}-A_{2} \exp (-s \tau)\right]^{-1} \\
\cdot B \mathcal{L}\{g[C x(t-\tau) ; \mu]\}
\end{aligned}
$$

or, equivalently,

$$
(\mathcal{L} e)(s)=-G(s ; \mu)(\mathcal{L} u)(s)
$$

where

$$
\begin{aligned}
e(t) & =-y(t)=-C x(t) \\
G(s ; \mu) & =C\left[s\left(\exp (-s \tau)+A_{0}\right)-A_{1}-A_{2} \exp (-s \tau)\right]^{-1} B \\
u(t) & =f[e(t-\tau) ; \mu]:=g[C x(t-\tau) ; \mu] .
\end{aligned}
$$




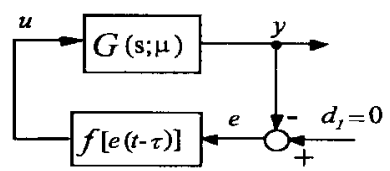

(a)

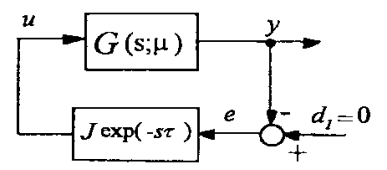

(b)

Fig. 1. (a) The nonlinear feedback path. (b) The linearized feedback system.

It should be pointed out here that the transfer function $G(s ; \mu)$ in the linear feedforward path depends on the frequency variable " $s$ " in a more complicated fashion as compared to that in the nondelayed version of the same frequency-domain setting [14].

The equilibrium solution of (3) $\hat{e}$ can be obtained by solving the following equation:

$$
G(0 ; \mu) f(e ; \mu)=-e
$$

and the linearization of the nonlinear feedback function about this equilibrium solution gives

$$
J \exp (-s \tau)=\left.\frac{\partial f}{\partial e}\right|_{e=\hat{e}} \exp (-s \tau) .
$$

The characteristic function of the linearized system is then given by

$$
\operatorname{det}[\lambda I-G(s ; \mu) J \exp (-s \tau)]=0 .
$$

The original nonlinear feedback system and its linearized configuration are given in Fig. 1(a) and (b), respectively, where " $d_{1}$ " can be a perturbation or a noise added to the model, but in this case, for simplicity, we use $d_{1}=0$.

Consider a periodic solution of (3) $e(t)$ and its $2 q$ th-order approximation

$$
e(t) \approx \hat{e}+\Re\left\{\sum_{k=0}^{2 q} E^{k} \exp (i k \omega t)\right\}
$$

where $\Re\{\cdot\}$ denotes the real part of a complex function (or number), $i=\sqrt{-1}, \omega$ is the fundamental frequency of the periodic solution, and $E^{k}$ is a complex number in the $k$ th harmonic of the expansion, which satisfies

$$
\begin{aligned}
E^{k} \exp \{i k \omega(t-\tau)\} & =E^{k} \exp (-i k \omega \tau) \exp (i k \omega t) \\
& =E_{d}^{k} \exp (i k \omega t)
\end{aligned}
$$

Hereafter, the subscript " $d$ " is used to indicate time-delayed quantities.

Fix the bifurcation parameter $\mu$ and, for simplicity, write $G(i \omega)=$ $\left.G(s ; \mu)\right|_{s=i \omega}$. Furthermore, notice from Fig. 1(a) that the harmonic balance can be performed by equating the output of the system (without time delay) with the input of the linear plant (with time delay), namely,

$$
E^{k}=-G(i k \omega) F_{d}^{k}, \quad k=0,1,2, \cdots, 2 q
$$

where $F_{d}^{k}=F_{d}^{k}\left(E_{d}^{0}, E_{d}^{1}, \cdots, E_{d}^{k}\right)$ are the Fourier coefficients for the expansion of the nonlinear function in the feedback path with time delay, as shown below. These delayed Fourier coefficients can be obtained from the existing formulas for Fourier coefficients without time delays [11, Appendix A] by including the delay factor "exp $(-i k \omega \tau)$." To briefly show the general procedure for such modification, expand the function $f[e(t-\tau)]$ in the Taylor series about the equilibrium solution $\hat{e}$ and then substitute the trial by delayed power terms up to a second-order HBA, namely, substitute

$$
\begin{aligned}
e(t-\tau) & =\hat{e}+\Re\left\{\sum_{k=0}^{2} E^{k} \exp (-i k \omega \tau) \exp (i k \omega t)\right\} \\
& =\hat{e}+\Re\left\{\sum_{k=0}^{2} E_{d}^{k} \exp (i k \omega t)\right\}
\end{aligned}
$$

into (7). We then obtain (9), shown at the bottom of this page, where - denotes the complex conjugate, $\otimes$ is the standard tensor product operator, $\rho_{0}, \rho_{1}$, and $\rho_{2}$ are higher order terms determined from higher order HBA's, and $D_{i}:=\left.\left(D^{i} f\right)\right|_{\hat{e}}$ is the multilinear $i$ th-order derivative operator evaluated at $\hat{e}$.

Next, let $E^{1}=v \theta$ in (9), where $v$ is the right eigenvector of $G(i \omega) J \exp (-i \omega \tau)$ associated with the eigenvalue $\hat{\lambda}$ (the eigenvalue $\lambda$ of (5) that is the closest to the critical point $(-1+i 0)$ and $\omega$ is sweeping onto the Nyquist contour), and $\theta$ is a measure of the amplitude of the oscillations of the periodic solution. We then have

$$
\begin{aligned}
E^{0}= & V_{02} \theta^{2} \\
= & -[I+G(0) J]^{-1} G(0) \frac{1}{2 !} D_{2}\left[\frac{1}{2} v \otimes \bar{v}\right] \theta^{2} \\
E^{2}= & V_{22} \theta^{2} \\
= & -[I+G(i 2 \omega) J \exp (-i 2 \omega \tau)]^{-1} G(i 2 \omega) \frac{1}{2 !} \\
& \cdot D_{2}\left[\frac{1}{2} v \otimes v \exp (-i 2 \omega \tau)\right] \theta^{2} \\
p_{1 d}(\omega, v, \tau):= & p_{1}(\omega, v) \exp (-i \omega \tau) \\
= & \left\{D_{2}\left(V_{02} \otimes v+\frac{1}{2} \bar{v} \otimes V_{22}\right)\right. \\
& \left.+\frac{1}{8} D_{3}(v \otimes v \otimes \bar{v})\right\} \exp (-i \omega \tau) .
\end{aligned}
$$

Finally, the complex number $\xi_{1 d}(i \omega)$, which is used to calculate the amplitude and frequency of the periodic solution, is obtained as

$$
\xi_{1 d}=-w^{\top} G(i \omega) p_{1 d}(\omega, v, \tau)
$$

where $w^{\top}$ is the left eigenvector of $G(i \omega) J \exp (-i \omega \tau)$ associated with the eigenvalue $\hat{\lambda}$, normalized according to $\left|w^{\top} v\right|=1$. To this

$$
\begin{aligned}
& E^{0}=-G(0) F_{d}^{0}=-G(0)\left\{J E^{0}+\frac{1}{2 !} D_{2}\left[\frac{1}{2} E^{1} \otimes \bar{E}^{1}\right]+\rho_{0}\right\} \\
& E^{1}=-G(i \omega) F_{d}^{1}=-G(i \omega)\left\{J E^{1} \exp (-i \omega \tau)+\frac{1}{2 !} D_{2}\left[2 E^{0} \otimes E^{1} \exp (-i \omega \tau)+\bar{E}^{1} \otimes E^{2} \exp (-i \omega \tau)\right]\right. \\
& \left.+\frac{1}{3 !} D_{3}\left[\frac{3}{4} E^{1} \otimes E^{1} \otimes \bar{E}^{1} \exp (-i \omega \tau)\right]+\rho_{1}\right\} \\
& E^{2}=-G(i 2 \omega) F_{d}^{2}=-G(i 2 \omega)\left\{J E^{2} \exp (-i 2 \omega \tau)+\frac{1}{2 !} D_{2}\left[\frac{1}{2} E^{1} \otimes E^{1} \exp (-i 2 \omega \tau)\right]+\rho_{2}\right\}
\end{aligned}
$$


end, using the characteristic-gain locus $\hat{\lambda}$ and the amplitude locus defined by

$$
L_{1 d}(\omega, \theta)=-1+\xi_{1 d}(\omega) \theta^{2}
$$

we can solve the following equation:

$$
\hat{\lambda}(i \omega ; \mu)=-1+\xi_{1 d}(i \omega) \theta^{2}
$$

for a solution pair $(\hat{\omega}, \hat{\theta})$. Finally, the approximate periodic solution $e(t)$ is obtained from (6) to (10).

Remark 1: In the above formulation, the function $f[e(t-\tau)]$, shown in Fig. 1(a), can have different time delays. For example, if

$$
f[e(t-\tau)]=f_{1}\left[e\left(t-\tau_{1}\right)\right]+f_{2}\left[e\left(t-\tau_{2}\right)\right]
$$

then we only need to modify (5) as follows:

$$
\operatorname{det}\left[\lambda I-G(s ; \mu)\left(J_{1} \exp \left(-s \tau_{1}\right)+J_{2} \exp \left(-s \tau_{2}\right)\right)\right]=0
$$

where $J_{1}=\left.\left(\partial f_{1} / \partial e\right)\right|_{e=\hat{e}}$ and $J_{2}=\left.\left(\partial f_{2} / \partial e\right)\right|_{e=\hat{e}}$. Equation (10) is then modified accordingly to

$$
\begin{aligned}
& E^{0}= V_{02} \theta^{2} \\
&=- {\left[I+G(0)\left(J_{1}+J_{2}\right)\right]^{-1} G(0) } \\
& \cdot \frac{1}{2 !}\left(D_{2,1}+D_{2,2}\right)\left[\frac{1}{2} v \otimes \bar{v}\right] \theta^{2} \\
& E^{2}=V_{22} \theta^{2} \\
&=- {\left[I+G(i 2 \omega)\left(J_{1} \exp \left(-i 2 \omega \tau_{1}\right)\right.\right.} \\
&\left.\left.+J_{2} \exp \left(-i 2 \omega \tau_{2}\right)\right)\right]^{-1} G(i 2 \omega) \\
& \times \frac{1}{2 !}\left(D_{2,1} \exp \left(-i 2 \omega \tau_{1}\right)+D_{2,2}\right. \\
& p_{1 d}\left(\omega, v, \tau_{1}, \tau_{2}\right)=\{\left(D_{2,1} \exp \left(-i \omega \tau_{1}\right)+D_{2,2}\right. \\
&\left.\cdot \exp \left(-i \omega \tau_{2}\right)\right)\left[V_{02} \otimes v+\frac{1}{2} \bar{v} \otimes V_{22}\right] \theta^{2} \\
&+\frac{1}{8}\left(D_{3,1} \exp \left(-i \omega \tau_{1}\right)\right. \\
&\left.\left.+D_{3,2} \exp \left(-i \omega \tau_{2}\right)\right)[v \otimes v \otimes \bar{v}]\right\} .
\end{aligned}
$$

Equation (11) remains the same, except that $p_{1 d}\left(\omega, v, \tau_{1}, \tau_{2}\right)$ is used to replace $p_{1 d}(\omega, v, \tau)$ therein.

Remark 2: The formulas given above can be easily extended to the fourth-, sixth-, and even eighth-order HBA's by following the simple rule stated in (8) (with higher order expansions) and the harmonic-balance principle specified by (7).

\section{Period-Doubling Bifurcations and Multiple Oscillations in Time-Delayed FeEdBack Systems}

In this brief, we use up to the sixth-order HBA by considering the fundamental equation

$$
\hat{\lambda}(i \omega ; \mu)=-1+\sum_{k=1}^{q=3} \xi_{k d}(\omega) \theta^{2 k}=: L_{k d} .
$$

Here, (13) ( $q=2$ gives the fourth-order HBA; $q=3$ sixth-order HBA) can be exactly solved without using any iterative computational scheme such as those employed in [11]. Although computationally this approach needs more central processing unit (CPU) time, it provides better approximations to the predicted periodic solutions. More importantly, it gives more insights to the PDB phenomenon in case the amplitude $\theta$ is less than one.

To predict possible PDB's, the algorithm works as follows (in three steps):
Step 1: Find an exact solution pair $(\hat{\omega}, \hat{\theta})$ of the amplitude locus $L_{1 d}(\omega, \theta, \mu)$ and $\hat{\lambda}(i \omega, \mu)$ for a given value of $\mu$ in the proximity of the Hopf bifurcation point $\left(\omega=\omega_{0}, \theta=0, \mu=\mu_{0}\right)$. Vary the value of $\mu$ and obtain a branch of periodic solutions from the Hopf bifurcation point until $\hat{\theta} \approx 1$ and $\mu=\mu_{\theta \approx 1}$.

Step 2: Find the second solution pair $\left(\hat{\omega}_{e}, \hat{\theta}_{e}\right)$ (if it exists) between $L_{1 d}(\cdot)$ and $\hat{\lambda}(\cdot)$ under the condition that $\hat{\theta}_{e}<1$ is in the same range of the variation of parameter $\mu$, i.e., $\mu_{0}<\mu<\mu_{\theta \approx 1}$ (or $\mu_{\theta \approx 1}<\mu<\mu_{0}$ ).

Step 3: Determine the value of $\mu$ (if it exists) in $\left[\mu_{0}, \mu_{\theta \approx 1}\right]$ (or $\left.\mu \in\left[\mu_{\theta \approx 1}, \mu_{0}\right]\right)$ at which $\hat{\omega}_{e} \approx \hat{\omega} / 2$. Label this value as $\mu_{p d}$. Then the resulting prediction is that a PDB is likely to occur at $\mu=\mu_{p d}$.

Remark 3: Since the Hopf bifurcation is a local phenomenon, convergence of the algorithm can be graphically checked provided that the approximations given by $L_{1 d}, L_{2 d}, L_{3 d}$, and so on are close enough, in the sense that the maximum (minimum) value of the predicted periodic solution does not change much (say, less than $10 \%$ ) if a second-order HBA is replaced by a fourth-order HBA in the calculation, a fourth-order HBA is replaced by a sixth-order HBA, and so on. This is true for a Hopf bifurcation, but since a PDB is a change in the global behavior of the cycle, we observe that the procedure works well if: 1) the PDB is inside the region of convergence of the series, i.e., $\theta \ll 1$ and 2 ) the procedure with higher order HBA's is still able to detect a second oscillatory frequency, which is near one-half of the regular frequency for the Hopf cycle. Comparing the prediction for different HBA's for the regular Hopf cycle and for the starting of the PDB, one gets a confidence of the existence of the PDB. Such a comparison can be easily accomplished numerically. Note that the point here is that in comparing the predictions obtained from different HBA's, we should reject those values that significantly differ. Note also that some equivalent measures such as the distortion index have already been proposed in the literature [15], for the purpose of discerning possible chaotic behaviors.

\section{Analysis of Two Time-Delayed Feedback Systems}

In this section, two systems with time delays are discussed: the TDCC and a neural-network model, where the former shows how the Hopf bifurcation formulas obtained in this section can be applied and how PDB's can be predicted, while the latter shows how the multiple oscillation feature of a time-delayed nonlinear system can be characterized, both from the developed graphical approach.

\section{Example 1: Bifurcations in the TDCC}

The dynamics of a scalar difference-differential equation can be very rich, as can be seen from the recent literature in electrical and electronic systems [8], [9], [16], [17]. The dynamics of the TDCC is one of such devices, which is described by (see [16])

$$
\begin{aligned}
\mu(1+\zeta) \frac{d x(t+1)}{d t}+\mu & (1-\zeta) \frac{d x}{d t}+x(t+1)+x(t) \\
& +(1+\zeta) g(x(t+1))+(1-\zeta) g(x(t))=0
\end{aligned}
$$

where $t$ is the time normalized with respect to the transmission-line delay $T ; g(\cdot)$ represents the nonlinear characteristic of the Chua's diode approximated by a cubic function $g(z)=\left(-m z+k z^{3}\right) / G$ with constants $m$ and $k$ normalized with respect to the conductance $G ; \zeta=Z G, \mu=C / G T, Z$ is the transmission-line characteristic impedance, and $C$ is the capacitance.

We study the TDCC dynamics with the variation of $\mu$ within the range [0.192303, 0.164], while the other parameters remain unchanged: $\zeta=0.7 / \sqrt{7}, m=4 / 5, k=2 / 45$, and $G=0.7$.

The transfer function $G(s, \mu)$ of the TDCC is

$$
G(s, \mu)=\frac{1}{[\mu(1+\zeta) \exp (s)+\mu(1-\zeta)] s+\exp (s)+1}
$$




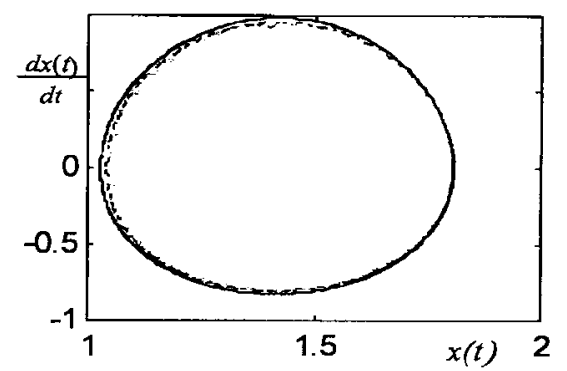

Fig. 2. Numerical simulation (solid line) and the prediction obtained by using $L_{1}$ approximation (dashed line) for $\mu=0.1875$.

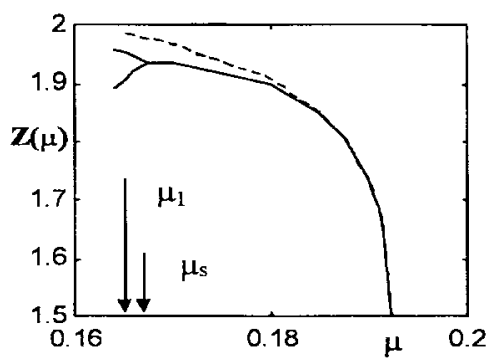

(a)

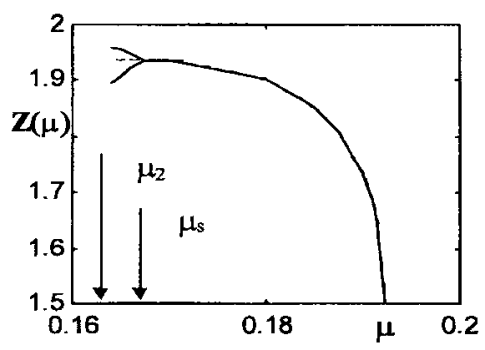

(b)

Fig. 3. (a) Simulation (solid line), $L_{1}$ prediction (dashed line). (b) Simulation (solid line), $L_{2}$ prediction (dashed line).

and the Jacobian $J$ of the nonlinear feedback function $f(e)=: g(x)$ after defining $e=-x$ is

$$
J=-(1+\zeta)\left[\frac{m}{G}-\frac{3 k \hat{e}^{2}}{G}\right] \exp (s)-(1-\zeta)\left[\frac{m}{G}-\frac{3 k \hat{e}^{2}}{G}\right]
$$

where $\hat{e}$ are the equilibrium solutions of the circuit obtained after solving (4), i.e., $\hat{e}_{1}=0$ and $\hat{e}_{2,3}= \pm 1.5$. We only analyze the system dynamics around the equilibrium solution $\hat{e}_{3}=-1.5$. Also, notice that we need to use Remark 1 with $\tau_{1}=-1$ and $\tau_{2}=0$.

Fig. 2 shows the plot of the true (numerically computed) results of a period-one stable limit cycle in the phase plane, with the $L_{1}$ and $L_{2}$ (we drop the subindex $d$ for simplicity) approximations, respectively, where $\mu=0.1875$. The $L_{2}$ approximation coincides exactly with the numerical simulation. Fig. 3(a) and (b) show the periodic branch diagram obtained by numerical simulations and with $L_{1}$ and $L_{2}$ approximations, respectively, under the variation of the system parameter $\mu$. The plotted function $Z(\mu)$ is the global maximum value of $x(t)$ when $\mu \in[0.167,0.192303]$, where the true orbit is a period-one limit cycle. At $\mu=0.167$, a PDB occurs, so $Z(\mu)$ becomes double-valued due to the occurrence of the local maximum values. It can also be seen that the $L_{2}$ approximation recovers the periodic branch more accurately than the $L_{1}$ approximation. However, for the $L_{1}$ approximation, the predicted

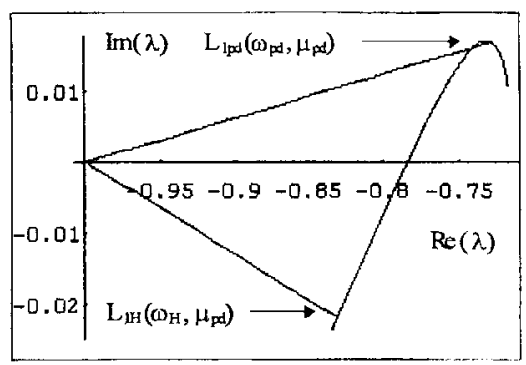

Fig. 4. $\quad L_{1}$ approximation and its intersections with $\hat{\lambda}$ at $\omega_{H}=1.9851$ and $\omega_{p d}=0.992917$ for $\mu=0.164$. In this case, $\omega_{p d} \approx \omega_{H} / 2$, so the system undergoes a period-doubling bifurcation.

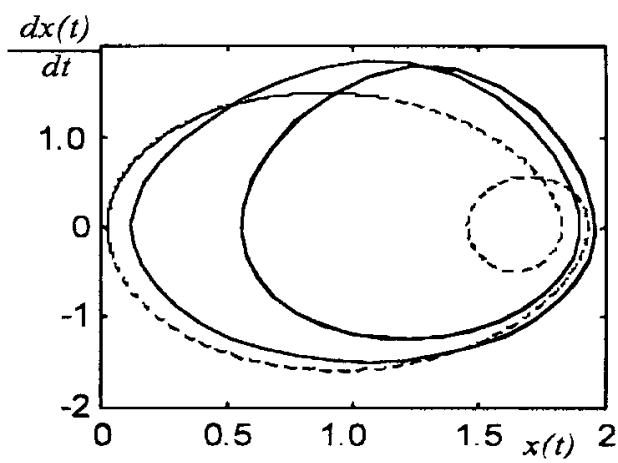

Fig. 5. Numerical simulation (sold line) and $L_{1}$ approximation for the predicted period-two cycle (dashed line) for $\mu=0.164$.

value of the bifurcation parameter for the PDB [marked as $\mu_{1}$ in Fig. 3(a)] is closer to the real value [denoted as $\mu_{s}$ in Fig. 3(a) and (b)] compared to the one predicted by the $L_{2}$ approximation [marked as $\mu=\mu_{2}$ in Fig. 3(b)].

Fig. 4 shows the curve of $\hat{\lambda}\left(\omega, \mu_{p d}\right)$, where $\mu_{p d}$ is the predicted value of $\mu$ for which the system undergoes a PDB. The intersection of $L_{1 H}\left(\omega, \mu_{p d}\right)$ with $\hat{\lambda}\left(\omega, \mu_{p d}\right)$ at the point $\hat{\lambda}\left(\omega_{H}, \mu_{p d}\right)$ provides a prediction of a period-one cycle of frequency $\omega_{H}$. The intersection of $L_{1 p d}\left(\omega, \mu_{p d}\right)$ with $\hat{\lambda}\left(\omega, \mu_{p d}\right)$ at the point $\hat{\lambda}\left(\omega_{p d}, \mu_{p d}\right)$, where $\omega_{p d} \approx$ $\omega_{H} / 2$ yields a prediction of a period-two cycle of frequency $\omega_{p d}$. Fig. 5 shows the predicted orbit compared to the true (numerically computed) result. It should be noticed that the predicted orbit almost reaches the origin point, which is an equilibrium solution. This indicates that the true value of $\mu$ for which a PDB occurs is likely to be greater than the one predicted here. When the true limit cycle touches the origin point, an interaction between the limit cycle and this unstable equilibrium point emerges. With respect to this circuit, such a phenomenon is a global behavior that appears after a PDB, which is known as a "double-scroll-like" attractor.

\section{Example 2: Multiple Oscillations of a Neural-Network Model}

The neural-network model considered here provides an interesting example for the detection of multioscillatory behaviors of nonlinear systems using the developed graphical-analysis method.

In this example, we show how the proposed algorithm can provide a clear picture about the coexistence of periodic solutions starting from other unstable modes, and how we can capture a large-amplitude limit cycle which exists from the beginning of the variation of the main bifurcation parameter by applying the graphical method.

Consider the neural-network model discussed in [12], described by

$$
\dot{x}(t)=A_{1} x(t)+A_{2} x(t-\tau)+B g[C x(t-\tau) ; \beta]
$$




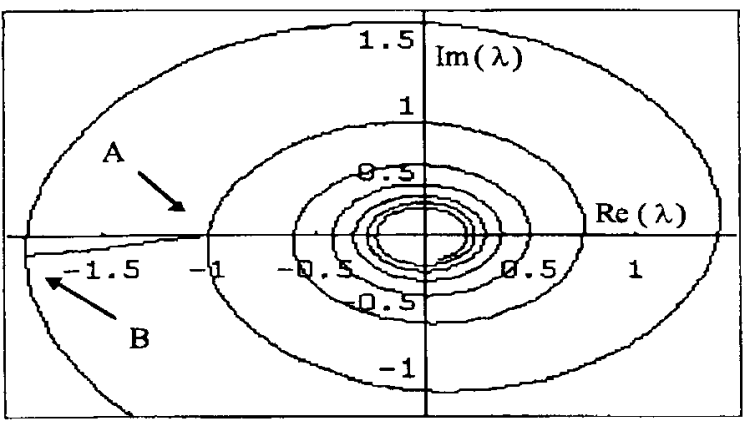

Fig. 6. $L_{3}$ approximation for two concentric orbits. $A$ of amplitude zero (at Hopf bifurcation). $B$ of large amplitude (predicted amplitude 2.88 , predicted frequency 0.37 ) and $\beta=1.772, \tau=4.265$.

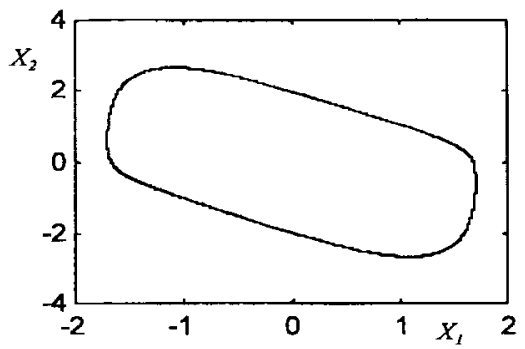

Fig. 7. Numerical simulation showing the existence of a stable periodic solution of amplitude 3.4 and frequency 0.3 , corresponding to the predicted cycle $B$ of Fig. 6 for $\beta=1.772, \tau=4.265$.

where

$$
\begin{aligned}
A_{1} & =\left[\begin{array}{rrr}
-1 & 0 & 0 \\
0 & -1 & 0 \\
0 & 0 & -1
\end{array}\right] \\
A_{2} & =0 \\
B & =\left[\begin{array}{ccc}
0 & 1 & 0 \\
-1.25 & 0 & 1 \\
1.25 & 1 & 0
\end{array}\right] \\
C & =\left[\begin{array}{lll}
1 & 0 & 0 \\
0 & 1 & 0 \\
0 & 0 & 1
\end{array}\right]
\end{aligned}
$$

and

$$
g[x(t-\tau)]:=f[e(t-\tau)]=\beta \tanh [e(t-\tau)]
$$

where $x(=:-e) \in R^{3}$ is the state vector, $\beta \in R$ is the bifurcation parameter, and $\tau \in R$ is the time delay constant.

Examine two regions in the space of the system parameters: 1) $1 \geq \beta \geq 0.96, \tau \geq 4.265$ and 2) $\beta \geq 1.7715, \tau \geq 4.265$.

In region 2), the system has a stable limit cycle due to a Hopf bifurcation at $\beta=0.96, \tau=4.265$, and $\omega_{0}=0.3898$. In this case, an eigenvalue of the linearized system crosses the point $-1+0 i$ when the frequency is sweeping onto the Nyquist contour.

In region 2), the system undergoes another Hopf bifurcation because another branch of the same eigenvalue locus crosses the point $-1+0 i$ again. The new cycle coexists with the one originated at $\beta=0.96$ and $\tau=4.265$, but the new limit cycle has a much smaller amplitude than the already existing one.

Fig. 6 shows the prediction of the two limit cycles ( $A$ is the smaller inner unstable cycle while $B$ is the larger stable one) using the $L_{3}$ approximation. Fig. 7 shows the true (numerically computed) outer limit cycle of larger amplitude. Here, the $L_{3}$ approximation actually has several more intersections with the characteristic locus (which are not shown in the figure), indicating the existence of other possible limit cycles for large values of $\theta$. However, a complete analysis of such possibilities requires the continuation of the periodic branches starting from different modes as well as their interactions. This is a complex task in this type of nonlinear time-delayed feedback systems, which we will not pursue any further in this brief. The developed graphical-analysis method nevertheless provides a useful and effective tool for prediction of PDB's starting close to Hopf bifurcation points, multiple oscillations, as well as local continuations of periodic solutions of nonlinear time-delayed feedback systems.

\section{CONCLUSIONS}

The graphical method developed in this paper for analyzing oscillations of nonlinear time-delayed feedback systems is effective not only for understanding periodic solutions, but also for predicting PDB's in the complex dynamical systems. This capability has been verified by correctly calculating the periodic solution branch starting from a Hopf bifurcation point and correctly predicting the PDB in the TDCC, and by correctly predicting the coexistence of two periodic solutions in a neural-network model. More applications of this frequency-domain approach to nonlinear time-delayed dynamical systems will be further investigated elsewhere.

\section{REFERENCES}

[1] J. G. Milton, A. Longtin, A. Beuter, M. C. Mackey, and L. Glass, "Complex dynamics and bifurcations in neurology," J. Theoretical Biol., vol. 138, pp. 129-147, 1989.

[2] J. Foss, A. Longtin, B. Mensour, and J. Milton, "Multistability and delayed recurrent loops," Phys. Rev. Lett., vol. 76, pp. 708-711, Jan. 1996.

[3] M. C. Mackey and L. Glass, "Oscillations and chaos in physiological control systems," Science, vol. 197, pp. 287-289, 1977.

[4] L. Glass and M. C. Mackey, "Pathological conditions resulting from instabilities in physiological control systems," Ann. N.Y. Acad. Sci., vol. 316, pp. 214-235, 1979.

[5] P. E. Rapp, R. A. Latta, and A. I. Mees, "Parameter-dependent transitions and the optimal control of dynamical diseases," Bull. Mather. Sci., vol. 50 , no. 3, pp. 227-253, 1988.

[6] J. Bélair, L. Glass, U. an der Heiden, and J. Milton, "Dynamical disease: Identification, temporal aspects and treatment strategies of human illness," Chaos, vol. 5, no. 1, pp. 1-7, 1995.

[7] S. A. Campbel, J. Bélair, T. Ohira, and J. Milton, "Complex dynamics and multistability in a damped harmonic oscillator with delayed negative feedback," Chaos, vol. 5, no. 4, pp. 640-645, Dec. 1995.

[8] J. Wu, "Global continua of periodic solutions to some difference differential equations of neutral type," Tôhoku Math. J., vol. 45, pp. 67-88, 1993.

[9] Y. Ueda, H. Ohta, and H. B. Stewart, "Bifurcations in a system described by a nonlinear differential equation with delay," Chaos, vol. 4, no. 1, pp. $75-83,1994$

[10] A. N. Sharkowsky, Yu. Maistrenko, P. Deregel, and L. O. Chua, "Dry turbulence from a time-delayed Chua's circuit," J. Circuits Syst. Comput., vol. 3, pp. 645-668, June 1993.

[11] J. L. Moiola and G. Chen, Hopf Bifurcation Analysis-A Frequency Domain Approach. Singapore: World Scientific, 1996.

[12] J. Bélair, S. A. Campbell, and P. van den Driessche, "Frustration, stability, and delay-induced oscillations in a neural network model," SIAM J. App. Math., vol. 56, no. 1, pp. 245-255, Feb. 1996.

[13] J. Wu, "Symmetric bifurcations of FDEs: Theory and applications to neural networks with memory," in Differential Equations and Control Theory, Z. Deng, Z. Liang, G. Lu, and S. Ruan, Eds. New York: Marcel Dekker, 1996, pp. 407-419.

[14] A. I. Mees and L. O. Chua, "The Hopf bifurcation theorem and its applications to nonlinear oscillations in circuits and systems," IEEE Trans. Circuits Syst., vol. CAS-26, pp. 235-254, Apr. 1979.

[15] R. Genesio and A. Tesi, "A harmonic balance approach for chaos prediction: Chua's circuit," Int. J. Bifurcation Chaos, vol. 2, no. 1, pp. 61-79, 1992.

[16] M. Gilli and G. M. Maggio, "Predicting chaos through an harmonic balance technique: An application to the time-delayed Chua's circuit," IEEE Trans. Circuits Syst. I, vol. 43, pp. 872-874, Oct. 1996.

[17] $\mathrm{H} . \mathrm{Lu}$ and $\mathrm{Z}$. He, "Chaotic behavior in first-order autonomous continuous-time systems with delay," IEEE Trans. Circuits Syst. I, vol. 43, pp. 700-702, Aug. 1996. 